\title{
Effect of Sleep Deprivation on the Inflammatory Response in Rats: A Pilot Study
}

\author{
Young Hyo Kim, MD, $\mathrm{PhD}^{1,2}$, Jieun Hong, $\mathrm{MS}^{1,2}$, Hyelim Park, $\mathrm{PhD}{ }^{1,2}$, \\ Ah-Yeoun Jung, MS ${ }^{1,2}$, Min Ki Lee, $\mathrm{MD}^{3}$, Ji Ho Choi, MD, $\mathrm{PhD}^{3}$ \\ 'Department of Otorhinolaryngology-Head and Neck Surgery, IInha Institute of Aerospace Medicine (IIAM), Inha University School of Medicine, \\ Incheon, Korea \\ ${ }^{3}$ Department of Otorhinolaryngology-Head and Neck Surgery, Soonchunhyang University College of Medicine, Bucheon Hospital, Bucheon, Korea
}

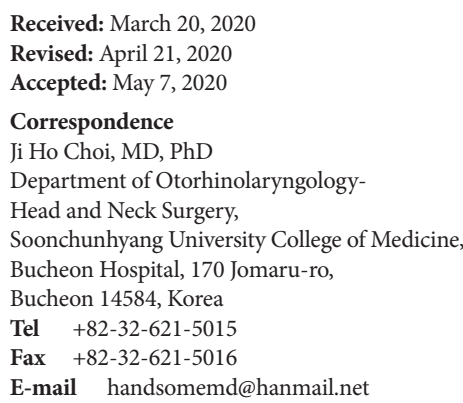

(c) This is an Open Access article distributed under the terms of the Creative Commons Attribution Non-Commercial License (https://creativecommons.org/licenses/by-nc/4.0) which permits unrestricted non-commercial use, distribution, and reproduction in any medium, provided the original work is properly cited.
We aimed to identify the association between sleep deprivation and inflammatory response. Fourteen-week old male Wistar rats were used randomly assigned to a sleep deprivation [sleep deprivation (SD) group, $\mathrm{n}=3$ ] or a control [healthy sleep (HS) group, $\mathrm{n}=3$ ] group. To induce sleep deprivation, we used a modified multiple platform method. We examined serum interleukin-1beta (IL-1 $\beta$ ), corticosterone, the number of inflammatory cells in broncho-alveolar lavage fluid (BALF), and the histopathology of the pulmonary parenchyma (peri-bronchiolar infiltration) between the SD and the HS groups. The SD group showed an increase in serum IL-1 $\beta$, corticosterone, the number of neutrophil and eosinophil in BALF, and peri-bronchiolar infiltration of eosinophils compared to the HS group. However, serum IL-1 $\beta$ and the number of inflammatory cells in BALF, except for corticosterone and eosinophil in lung parenchyma, were statistically insignificant. We suggest that SD may be associated with worsening of allergic inflammatory response.

Sleep Med Res 2020;11(1):53-57

Key Words Sleep deprivation, Allergy, Inflammation, Histopathology.

\section{INTRODUCTION}

Sleep accounts for approximately one-third of human life and has been an essential part of human survival [1]. However, sleep has been regarded as a passive state or physiological phenomenon, and the significance of sleep has not been recognized. In particular, appropriate sleep duration is significant to maintaining vigilance during waking. Sleep deficiency is clinically associated with a variety of sleep disorders, such as insomnia and insufficient sleep syndrome [2]. Also, short sleep duration can increase the risk of various consequences, including cardiovascular disorders, metabolic diseases, and mortality [3-5].

An allergy is characterized by overactive responses of the immune system resulting from a specific allergen [6]. Allergic disease is clinically related to several chronic inflammatory disorders such as allergic rhinitis, atopic dermatitis, and asthma [7].

A few studies have investigated the link between sleep deprivation and allergic inflammatory response in animal models [8,9]. However, it is not fully known about the relationship between sleep deprivation and allergic inflammatory response. We hypothesize that insufficient sleep could cause the deterioration of the allergic reaction. Thus, the purpose of this study was to determine the possibility that sleep deprivation is associated with inflammatory response in rats. 


\section{METHODS}

\section{Animals}

Male Wistar rats of age 14 weeks were used for the experiment and randomly assigned to a sleep deprivation [sleep deprivation (SD) group, $\mathrm{n}=3$ ] or a control group [healthy sleep (HS) group, $\mathrm{n}=3$ ). They were in a controlled environment, with a 12-hour light-dark cycle and free access to food and water. All rats used in the study were handled according to the protocol approved by the Institutional Animal Care and Use Committee of Inha University Hospital (INHA 181207-605).

\section{Animals \& Protocol for Sleep Deprivation}

A modified multiple platform model was used to induce sleep deprivation (Fig. 1) [10]. To be brief, we installed several $6.5 \mathrm{~cm}$ diameter cylinders inside the cage and filled the water up to $1 \mathrm{~cm}$ below the height of the cylinder. The rats could move freely on the platform and to feed and intake water. However, when rats sleep, they lose their center on the platform and fall into the water so that we could induce rat sleep deprivation. We also set up a 'platform cage control group'. In this group, we used the same cage and filled the water equally up to $1 \mathrm{~cm}$ below the cylinder. However, because the platform was $14 \mathrm{~cm}$ in diameter (much

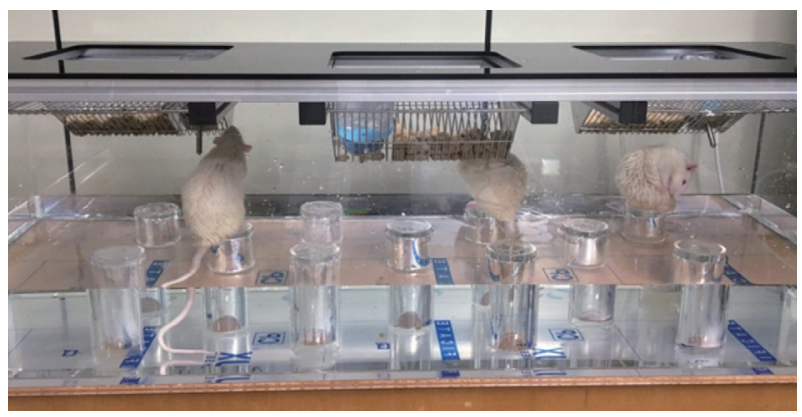

Fig. 1. A modified multiple platform model to induce sleep deprivation to rats. broader compared to the SD group), the rats could sleep on the platform. We exposed each rat continuously for 72 hours under SD or HS conditions and sacrificed them immediately after exposure. Before and after 72 hours of HS or SD exposure, we measured the weight of each rat and calculated the change.

\section{Measurement of Serum Levels of Interleukin-1Beta and Corticosterone}

Immediately after the exposure to SD or HS, the rats were sacrificed. We collected blood by the aortic puncture technique from the abdominal aorta. We then centrifuged the whole blood for 30 minutes at $4^{\circ} \mathrm{C}, 13,000 \times \mathrm{g}$. The supernatant was immediately stored at $-80^{\circ} \mathrm{C}$ until future analysis. After diluting the serum to 1:50, we measured the serum level of interleukin-1beta (IL-1 $\beta$, R\&D Systems, MN, USA) and corticosterone (Abcam, Cambridge, UK) using enzyme-linked immunosorbent assay, according to the protocol provided by the manufacturer.

\section{Collection of Broncho-Alveolar Lavage Fluid}

To collect broncho-alveolar lavage fluid (BALF), we cannulated the trachea using the polyethylene tube. We lavaged the lung with Hank's balanced salt solution (Thermo Fisher Scientific, Waltham, MA, USA). We centrifuged the lavage fluid at $4^{\circ} \mathrm{C}$ for 15 minutes, at $3,000 \times \mathrm{g}$. The pellet was immediately suspended in physiologic saline for cell counting. We determined the total cell numbers in duplicate using a hemocytometer. We centrifuged an aliquot of $100-200 \mu \mathrm{L}$ in a Cytospin 2 cytocentrifuge (Shandon Scientific, Pittsburgh, PA, USA). We determined the differential counts of eosinophils, neutrophils, and lymphocytes from the centrifuged preparation, which were stained using the Diff-Quik stain kit (Sysmex Corp., Kobe, Japan) by counting $>500$ cells from each sample at $\times 200$ of magnification. The representative photograph of BALF neutrophils are shown in Fig. 2.

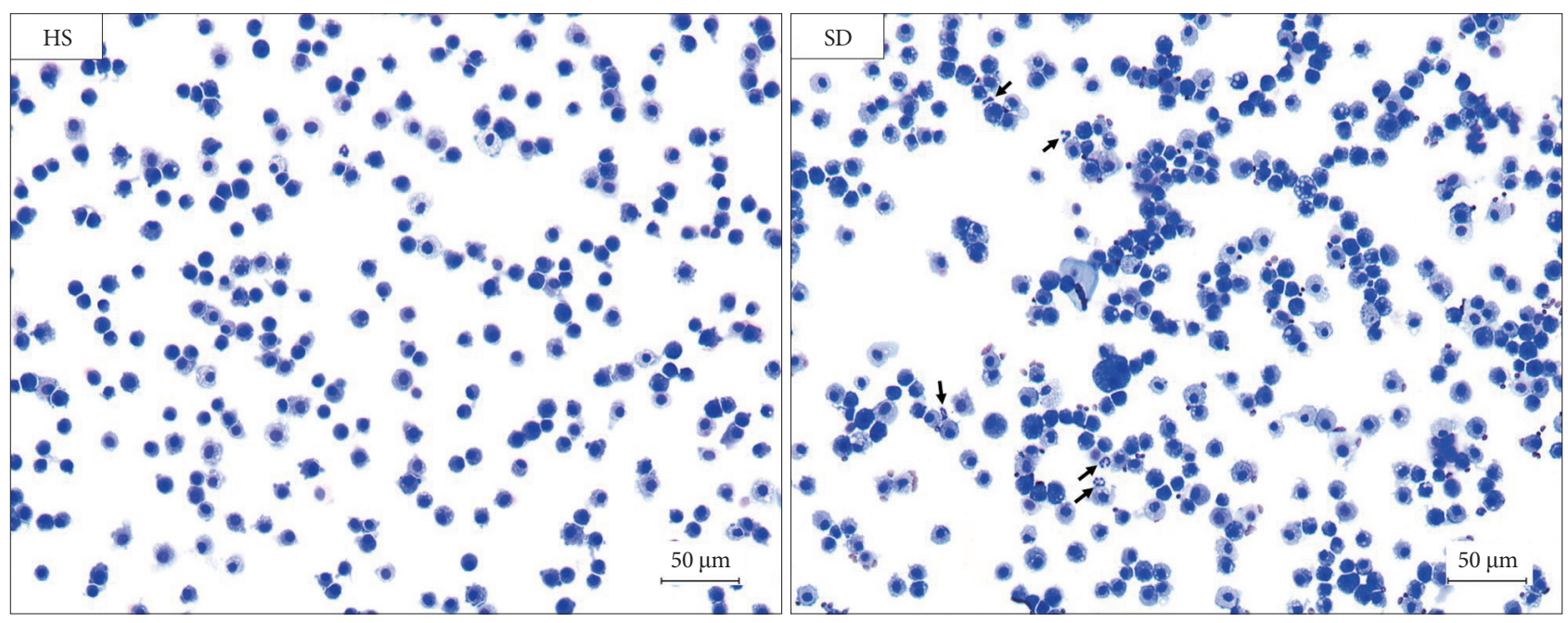

Fig. 2. The representative photograph of the broncho-alveolar lavage fluid cells (Diff-Quik staining, $\times 200$ of magnification). Arrows: neutrophils. HS: healthy sleep, SD: sleep deprivation. 


\section{Histopathologic Examination}

We fixed tissue specimens of the lung in $4 \%$ paraformaldehyde for 24 hours. We washed the lung tissues using deionized water, and then, embedded them in paraffin. We stained the tissue sections (of $3 \mu \mathrm{m}$ thickness) using hematoxylin \& eosin. We examined the total counts of eosinophils in pulmonary parenchyma (peri-bronchiolar infiltration).

\section{Statistical Analysis}

We used the SPSS 22.0 program (IBM Corp., Armonk, NY, USA) for the statistical analysis. We adopted the nonparametric tests, such as the Kruskal-Wallis test to compare the change of body weight, serum IL-1, corticosterone, the number of inflammatory cells in BALF and the counts of eosinophils in lung parenchyma. A p-value $<0.05$ was statistically significant.

\section{RESULTS}

After 72 hours, rats in the HS group had some gain of body weight $(4.3 \pm 2.0 \mathrm{~g})$. Conversely, rats belonging to the $\mathrm{SD}$ group showed significant decrease in weight after sleep deprivation $(-20.2 \pm 2.5 \mathrm{~g}, \mathrm{p}=0.005)$.

Compared with the HS group, the SD group showed increase in serum IL- $1 \beta$ and corticosterone, and the latter showed a statistically significant difference ( $\mathrm{p}=0.002$ for corticosterone) (Fig. 3).

When comparing the number of inflammatory cells in BALF of the $\mathrm{HS}$ group and the $\mathrm{SD}$ group, the SD group showed more neutrophil and eosinophil count. However, this did not have statistical significance ( $p>0.05$ for eosinophil, neutrophil and lymphocyte) (Fig. 4).

Comparing the difference of the pulmonary parenchyma according to the sleep patterns between the groups, the SD group showed increased peri-bronchiolar infiltration of eosinophils (Fig. 5). Comparing the count of eosinophils in $1 \mathrm{~mm}^{2}$ of lung parenchyma, we could note that the SD group had significantly increased infiltration of eosinophils compared to the HS group (the HS group: $4.5 \pm 0.9 \times 10^{3} / \mathrm{mm}^{2}$ versus the SD group: 16.7 $\pm 5.2 \times 10^{3} / \mathrm{mm}^{2}, \mathrm{p}=0.017$ ).

\section{DISCUSSION}

Various allergy-related parameters including IL-1 $1 \beta$, corticosterone, the number of inflammatory cells in BALF, and the histopathology of the pulmonary parenchyma were evaluated in this study to determine the relationship between sleep deprivation and allergic inflammation through animal experiments.
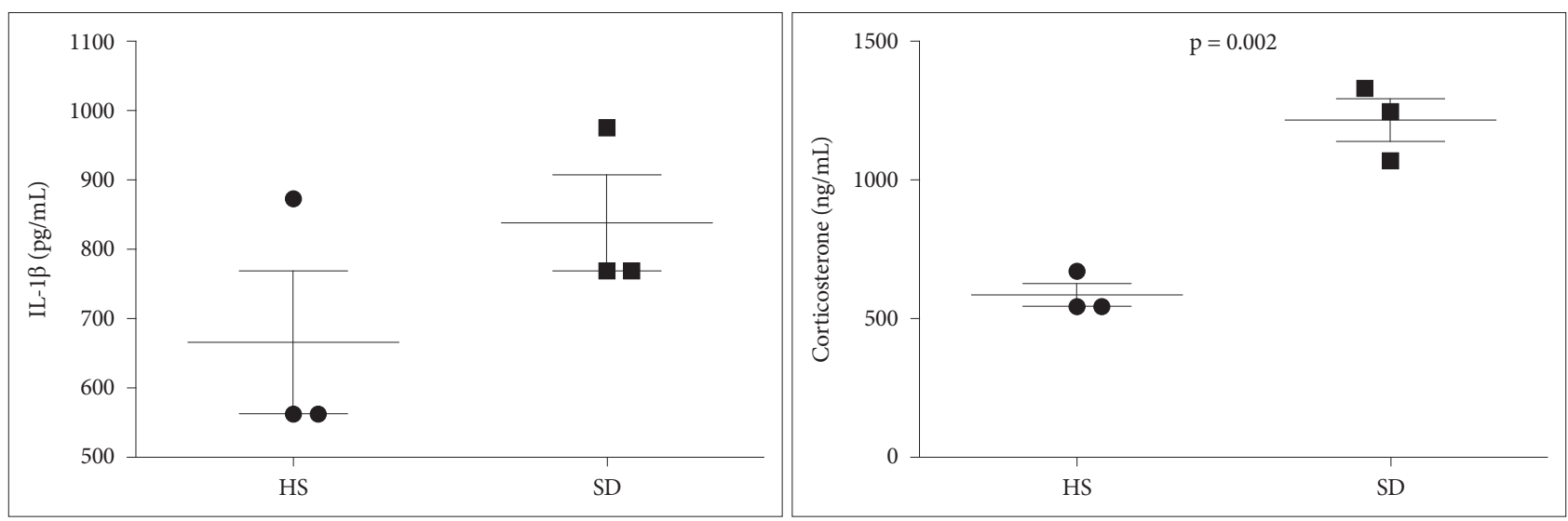

Fig. 3. Comparison of serum IL-1 $\beta$ and corticosterone between the HS and the SD groups. The SD group showed increase in serum IL-1 $\beta$ and corticosterone compared to the HS group. There was statistically significant increase in corticosterone levels $(p=0.002)$. IL-1 $\beta$ : interleukin-1beta, HS: healthy sleep, SD: sleep deprivation.
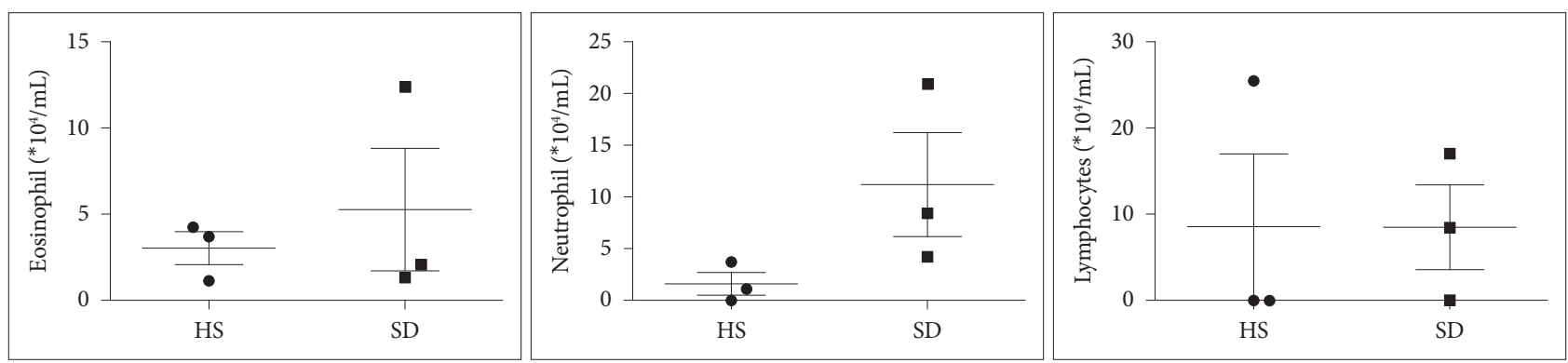

Fig. 4. Comparison of the number of inflammatory cells in BALF between the HS group and the SD group. The SD group showed increase in neutrophil counts compared to the HS group. However, there were no significant differences in eosinophil, neutrophil and lymphocyte counts. BALF: broncho-alveolar lavage fluid, HS: healthy sleep, SD: sleep deprivation. 


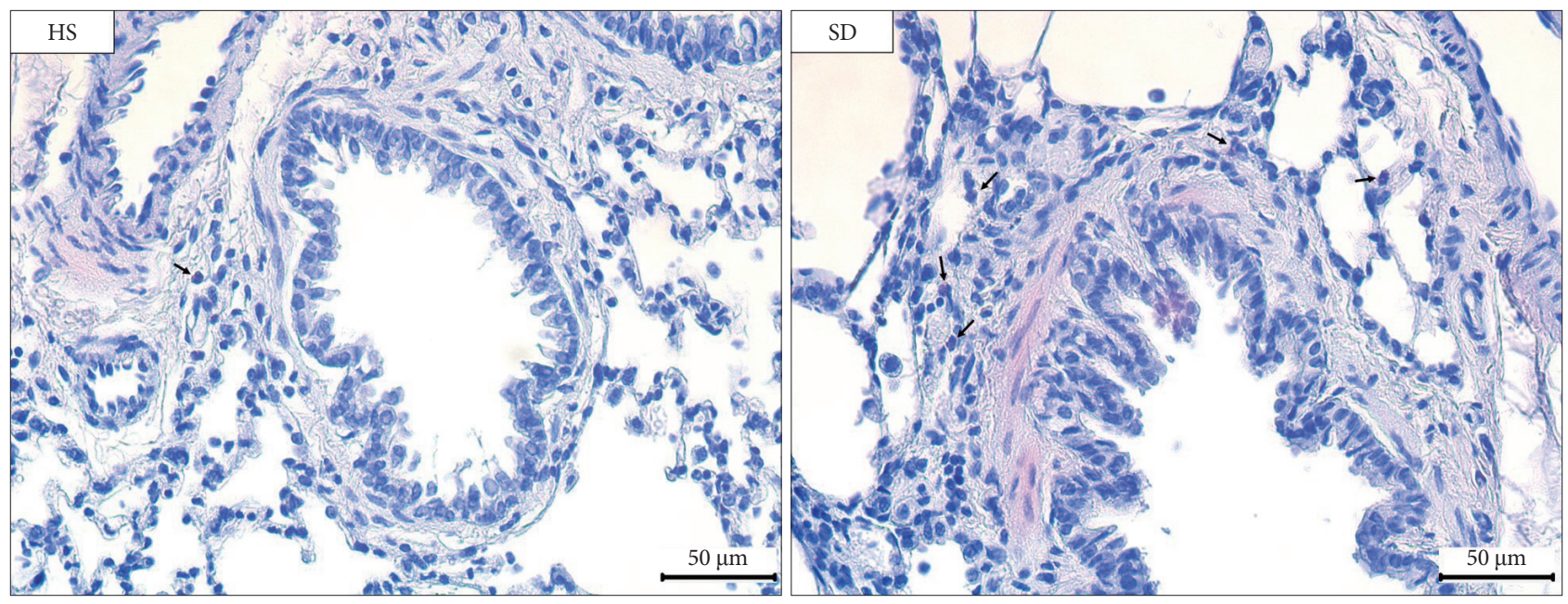

Fig. 5. Histopathology of the pulmonary parenchyma in the HS group and the SD group (Hematoxylin \& Eosin staining, $\times 400$ of magnification). The SD group showed increased peri-bronchiolar infiltration of eosinophils compared to the HS group $(p=0.017)$. HS: healthy sleep, SD: sleep deprivation.

The outcomes of this study provided that the SD group had increase in serum IL-1 $\beta$, corticosterone, the number of inflammatory cells (e.g., neutrophil and eosinophil) in BALF, and peribronchiolar infiltration of inflammatory cells compared to the HS group. Diverse mechanisms may be presumed for sleep deprivation to affect the exacerbation of allergic reactions. As is well known, sleep deprivation is associated with chronic inflammation, nervous or hormonal imbalance, and immunologic change $[8,9,11,12]$. These can cause or worsen allergic responses. Irie et al. [8] evaluated the influence of REM sleep deprivation using the classical platform model on immediate and late asthmatic responses in a rat model of bronchial asthma. They found that REM sleep is related to nocturnal asthma, probably because of changes in sympathetic modulation. Nunes et al. [9] examined the effect of sleep deprivation on allergen-induced airway inflammation in an ovalbumin (OVA)-sensitized mice model and reported that there were severe lung inflammation in the sleepdeprived OVA-sensitized mice group compared to the control group. In detail, their results indicated that sleep deprivation is related to elevated IL-17 levels, pulmonary neutrophilia, and resistance to corticosteroid therapy. However, in this study, there were no significant differences in serum IL-1 $\beta$ and the number of inflammatory cells in BALF except for corticosterone between the two groups. These results are attributable to several limitations. It is mainly thought to be because of the small sample size and the period of sleep deprivation. Jung et al. [11] assessed if auditory functions such as hearing levels are influenced by the sleep deprivation in an animal model and established that sleep deprivation may induce increases of inflammatory cytokines and the destruction of cochlea hair cells, causing hearing loss. In their study, a significant elevation in IL- $1 \beta$ levels was found in the sleep deprivation nine days group $(n=12)$ compared to the control group ( $n=15)$, in future studies, it is necessary to use more samples or to apply varied sleep deprivation periods. Our result showed that there was a significant increase in the corticosterone levels in the SD group compared to the HS group. These outcomes are consistent with those of many types of research that estimate serum corticosterone concentrations in rats with sleep deprivation. Choi et al. [12] investigated the association between sleep deprivation and the male reproductive system, including blood steroid hormone levels, and found that corticosterone levels significantly elevated in the sleep deprivation in the four days and seven days groups compared to the control group.

One of the main disadvantages of this study was that the number of animals used in this experiment was too small (only three rats per group). In the modified platform model, we used the rats live for 72 hours by moving between platforms. However, if we have too many rats in a platform model, the cage is too crowded, and rats cannot move freely, which is another stressor. In our previous research, we found that if too many rats were placed in the platform model, there could be various problems such as drowning. Since this study has the characteristics of a pilot study, in the future, we will endeavor to confirm the results of this study and deduce more meaningful conclusions by repeating experiments several times.

Previous studies show that stress can increase airway inflammation [13]. The mechanism may be the endogenous circulating corticosterone significantly modulate immune cell differentiation and function. For example, a stress-induced hormone promotes pro-allergic Th2-type responses and the level of corticosterone in plasma inhibits the IL-12/interferon $-\gamma$ regulatory axis [14-16].

In conclusion, it is crucial to reveal the association between sleep and allergy in modern society, where sleep duration is gradually decreasing, and allergic diseases are increasing. The results of this pilot study indicate that sleep loss may be associated with deterioration of allergic inflammatory response. Further study using more subjects is required. 


\section{Acknowledgments}

This study was supported by the KSSM (Korean Society of Sleep Medicine) Research Fund and by the Ministry of Education, Science, and Technology through the National Research Foundation of Korea (NRF2018R1A6A1A03025523)

\section{Conflicts of Interest}

The authors have no financial conflicts of interest.

\section{Authors' Contribution}

Conceptualization: Choi JH. Data curation: Kim YH. Formal analysis: Hong J, Jung AY. Funding acquisition: Choi JH, Kim YH. Investigation: Jung AY, Park H. Supervision: Choi JH, Kim YH. Writing-original draft: Kim YH. Writing_review \& editing: Choi JH, Lee MK.

\section{REFERENCES}

1. Siegel JM. Clues to the functions of mammalian sleep. Nature 2005; 437:1264-71.

2. American Academy of Sleep Medicine. International classification of sleep disorders. 3rd ed. Darien, IL: American Academy of Sleep Medicine, 2014.

3. Cappuccio FP, Cooper D, D'Elia L, Strazzullo P, Miller MA. Sleep duration predicts cardiovascular outcomes: a systematic review and meta-analysis of prospective studies. Eur Heart J 2011;32:1484-92.

4. Ju SY, Choi WS. Sleep duration and metabolic syndrome in adult populations: a meta-analysis of observational studies. Nutr Diabetes 2013; 3:e65.

5. Cappuccio FP, D’Elia L, Strazzullo P, Miller MA. Sleep duration and all-cause mortality: a systematic review and meta-analysis of prospective studies. Sleep 2010;33:585-92.
6. Milgrom $\mathrm{H}$, Huang $\mathrm{H}$. Allergic disorders at a venerable age: a mini-review. Gerontology 2014;60:99-107.

7. Pols DH, Wartna JB, Moed H, van Alphen EI, Bohnen AM, Bindels PJ. Atopic dermatitis, asthma and allergic rhinitis in general practice and the open population: a systematic review. Scand J Prim Health Care 2016;34:143-50.

8. Irie M, Nagata S, Endo Y, Kobayashi F. Effect of rapid eye movement sleep deprivation on allergen-induced airway responses in a rat model of asthma. Int Arch Allergy Immunol 2003;130:300-6.

9. Nunes JOF, Apostolico JS, Andrade DAG, Ruiz FS, Fernandes ER, Andersen $\mathrm{ML}$, et al. Sleep deprivation predisposes allergic mice to neutrophilic lung inflammation. J Allergy Clin Immunol 2018;141:10181027.e4.

10. Machado RB, Hipólide DC, Benedito-Silva AA, Tufik S. Sleep deprivation induced by the modified multiple platform technique: quantification of sleep loss and recovery. Brain Res 2004;1004:45-51.

11. Jung JH, Kim M, Lee SJ, Lee E, Lee SA, Lee JD, et al. Effect of sleep deprivation on hearing levels in rats. Int J Pediatr Otorhinolaryngol 2018; 112:169-75.

12. Choi JH, Lee SH, Bae JH, Shim JS, Park HS, Kim YS, et al. Effect of sleep deprivation on the male reproductive system in rats. J Korean Med Sci 2016;31:1624-30.

13. Joachim RA, Quarcoo D, Arck PC, Herz U, Renz H, Klapp BF. Stress enhances airway reactivity and airway inflammation in an animal model of allergic bronchial asthma. Psychosom Med 2003;65:811-5.

14. Elenkov IJ. Glucocorticoids and the Th1/Th2 balance. Ann N Y Acad Sci 2004;1024:138-46.

15. Montoro J, Mullol J, Jáuregui I, Dávila I, Ferrer M, Bartra J, et al. Stress and allergy. J Investig Allergol Clin Immunol 2009;19 Suppl 1:40-7.

16. Agarwal SK, Marshall GD Jr. Stress effects on immunity and its application to clinical immunology. Clin Exp Allergy 2001;31:25-31. 